\title{
Gain radial distribution of copper bromide lasers
}

\author{
S. Behrouzinia \\ 14399511-13, Tehran, Iran \\ Email addresses: $\underline{\text { sbehrouzi@aeoi.org.ir }}$
}

Photonics and Quantum Technologies Research School, Nuclear Science and Technology Research Institute, PO Box

Received August 2020

Received in revised: August 2020

Published: Sept 2020

\begin{abstract}
The paper presents the experimental results on how the tube bore of copper bromide laser influence the gain radial distribution. A pair of copper bromide lasers in an oscillator- amplifier configuration was operated to measure and compare the small-signal gains of different laser tube bores. The gain radial distributions were measured in different size of amplifier tube bores. It was shown that, by changing of the amplifier tube bore, the gain radial distribution was changed, similar to that of temperature profile, but the average of radial gains through cross-section are the same in different laser tube bores.
\end{abstract}

Keywords: Copper bromide laser, Gain radial distribution, Tube bore.

C2020 The Authors. Published by Fundamental Journals. This is an open access article under the CC BY-NC https://creativecommons.org/licenses/by-nc/4.0/

https://doi.org/10.14331/ijfps.2020.330138

\section{INTRODUCTION}

Copper bromide lasers (CBL), as a kind of halide lasers, can produce the wavelengths of $510.6 \mathrm{~nm}$ (green) and $578.2 \mathrm{~nm}$ (yellow) in visible region, similar to that of copper vapor lasers (CVL); therefore the CBL has the same attractive applications of CVLs in different areas of science and technology. The important advantage of a CBL compared with the CVL is operating at lower temperature $\left(\sim 510^{\circ} \mathrm{C}\right)$ and having higher beam quality, which is better suited to many applications such as brightness amplifiers in visualization system-laser monitors (Fedor Aleksandrovich Gubarev \& Klenovskii, 2016; Kulagin, Torgaev, \& Evtushenko, 2020). Such system have been used to study the high-temperature processes and modification of materials, and gain plays as a key role in this manner (Li, Ilyin, Gubarev, Mostovshchikov, \& Klenovskii, 2018; Trigub, Platonov, Evtushenko, Osipov, \& Evtushenko, 2017).

In addition, some works have been studied on spatial-temporal gain distribution on CBL (Evtushenko, 2015; Fedor Alexandrovich Gubarev, Trigub, Klenovskii, Li, \&
Evtushenko, 2016). On the other hand, the master oscillatorpower amplifier (MOPA) array has been used extensively in order to determine the small-signal gain $\left(\mathrm{g}_{0}\right)$, and saturation intensity $\left(E_{S}\right)$, as laser amplifying parameters, in metal vapor lasers (S Behrouzinia, Khorasani, Kazemi, \& Mashayekhi, 2011; S Behrouzinia, Sadighi-Bonabi, Parvin, \& Zand, 2004; Saeid Behrouzinia, Sadighi, \& Parvin, 2003; S. M. Lima, Behrouzinia, \& Khorasani, 2019; S. M. Lima et al., 2017; S. M. Lima, Behrouzinia, S., Salem, M.K., Elahei, M., Khorasani, K.,Dorranian, D. , (2017); Nezhad, Sajad, Behrouzinia, Salehinia, \& Khorasani, 2010).

In our previous work, the independencies of the amplifying parameters of CBL on the different amplifier tube length with identical diameter has been investigated, individually. It has been shown that, by changing the amplifier tube length, the amplifying parameters remain nearly constant without any remarkably changing and moreover, the gain radial distribution has been studied (S. M. Lima et al., 2019). 
In other works, the gain profile in large and medium bores of CBLs tubes, through one pass gain (Fedor Alexandrovich Gubarev, Troitskiy, Trigub, \& Sukhanov, 2011) and double pass gain (F. Gubarev, Li, Klenovskii, \& Shiyanov, 2016) methods have been investigates, so.

In this work, the CBL MOPA has been employed to obtain the behavior of gain radial distributions on different laser bore tubes. It is shown that, the gain radial distribution of CBLs is dependent on tube's bore, but the average of radial gains among cross-section of tubes are the same in different laser tube bores.

\section{EXPERIMENTAL SETUP}

Six cylindrical tubes with different volumes in three class of region; bores of 1.8- (small bore, 2\#), 3- (medium bore, 2\#) and $5 \mathrm{~cm}$ (large bore, $2 \#$ ) with these's optimum corresponding lengths of $25-, 50-$ and $100 \mathrm{~cm}$ have been employed to investigate the effect of the tube's bore on the gain radial distribution. For measuring of the $\mathrm{g}_{0}$, which leads to gain, we used the Hargrov's model (S. M. Lima et al., 2019). Fig.1. illustrates schematic representation of CBL MOPA which has been arranged for our main experiments. The master oscillator has been operated with a critical resonator constructed by a flat dielectric coated with high-reflection coefficient of about $98 \%$ as a back mirror, and uncoated flat quartz with reflection coefficient of about $4 \%$ as the output coupler. The input and output powers of the amplifier are measured by two Molectron ${ }^{\mathrm{TM}}$ PM500D power- meters, simultaneously. Two high-voltage probes P6015 and a Tektronix ${ }^{\text {TM }}$ TDS-1250 oscilloscope have been used for controlling of the delay times. An independent electrical power source and its corresponding pulse generator have driven each laser head. Two subsequent identical electrical discharges are produced whose relative delay time can be controlled manually by triggers (synchronization process).

The attenuation of the oscillator beam by some neutral density filters, which is subsequently injected into the amplifier, provides data on the overall amplification ratio, in order to determine the amplification parameters of amplifier, especially $g_{0}$ here.

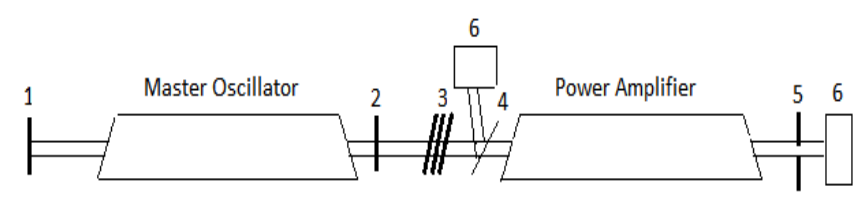

Fig.1. CBL MOPA configuration for gain measurement; 1, 2resonator cavity of the master oscillator;3- neutral density filters; 4- partial reflector; 5- circular diaphragm; 6- powermeters.

The optimum pulse repetition frequencies are 18-, 13-, and 4.5 $\mathrm{kHz}$ in tube bores of $1.8-, 3-$, and $5 \mathrm{~cm}$, respectively. The Helium gas is used as buffer gas at optimum pressure of $\sim 12$ torr. It has been tried to make more uniformity of the oscillator beam, as possible, through adding amount of $1-3 \% \mathrm{H}_{2}$ in buffer gas, depending on the tube geometry. Also, the radial distribution of the oscillator beam close to uniform pattern is possible to provide with good optimization of the operating conditions of the active elements. A series of diaphragms contains circular grooves with different radius are used perpendicular to the output of amplifier, individually, to measuring the local radial output power, Fig.2.

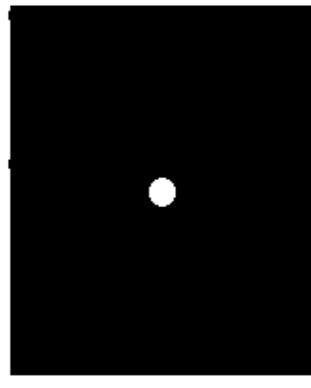

a

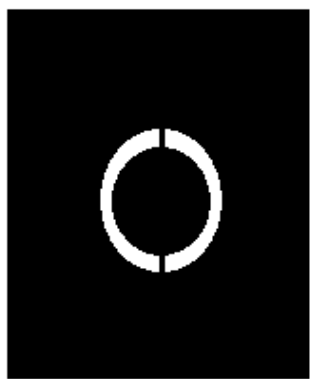

d

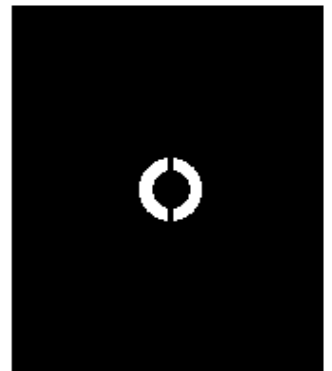

b

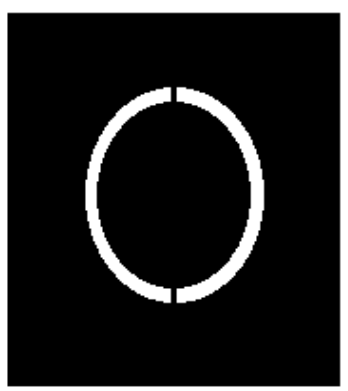

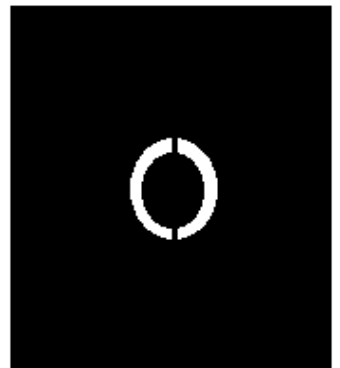

c

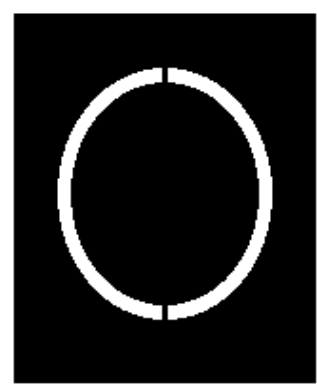

g

Fig.2. Diaphragms with different radius of circular grooves; (a) a hole with diameter of $6 \mathrm{~mm}$ in the center, which coincide by the tube axis. (b)-(g) a circular groove with inner diameters of 6, 12, 18, 24, 30 and 36mm and corresponding outer diameters of $12,18,24,30,36$ and $42 \mathrm{~mm}$, respectively.

The first diaphragm has a hole which is adjusted in the center of the diaphragm (a), and the other diaphragms have ring holes with different radius. Diaphragms a-c are used in small-bore tube, a-e are used in medium one, and a-g are used in large one, individually. The central hole is adjusted in front of the amplifier axis and the last one is alignment near the tube wall. 


\section{RESULTT AND DISCUSSION}

Since the Doppler broadening is sufficiently greater than the pressure broadening line-width for halide lasers like CVL, the amplifying medium is assumed to be an inhomogeneous broadened gain medium.

The amplifying parameters of $\mathrm{g}_{0}\left(\mathrm{~cm}^{-1}\right)$ and $\mathrm{E}_{\mathrm{S}}\left(\mu \mathrm{J} / \mathrm{cm}^{2}\right)$ can be calculated with a least-squares fitting of the experimental data into Hargrove equation (S. M. Lima et al., 2019).

Fig. 3 shows the small-signal gains radial distributions for tube bores of 1.8- (a), 3- (b), and 5cm (c), respectively, by using diaphragms. In Fig.3, zero position along the horizontal coordinate axis corresponds to the axis of tube.

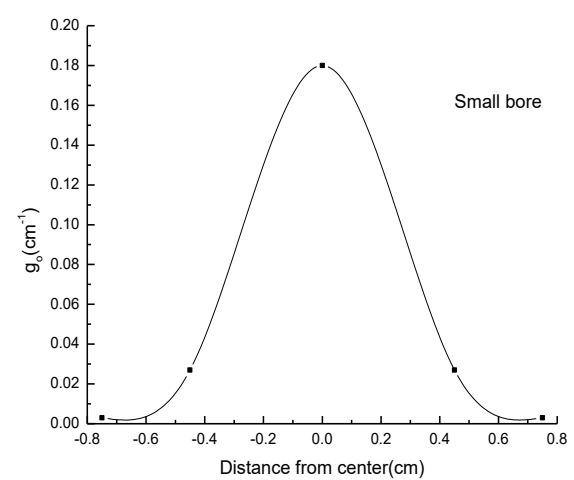

(a)

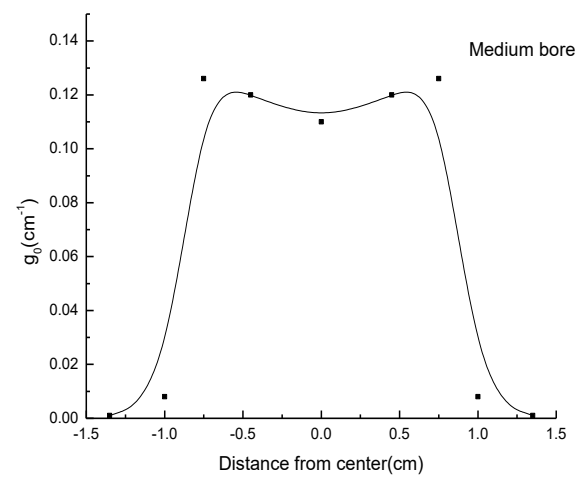

(b)

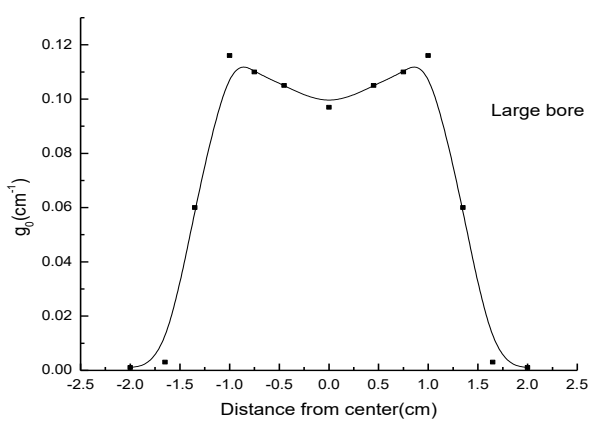

(c)

Fig.3. Small-signal gains radial distributions; bores of small (a), medium (b), and large (c) tube.
It should be noted that, the average of $\mathrm{g}_{0} \mathrm{~s}$ is the same and equal to about $0.07 \mathrm{~cm}^{-1}$ for all tubes. The $\mathrm{g}_{0}$ in the axial region of small tube (a) reaches to its maximum value, and then dropped suddenly at distances far from the center, and the gain profile has a Gaussian's shape.

The profiles of $\mathrm{g}_{0}$ in the medium and large bores (b) and (c) have a dip at the center of tube, and at distance far from the center, the $\mathrm{g}_{0}$ is increases and reach to its maximum value, symmetrically and then dropped suddenly at distance more far from the center. The reduction of $\mathrm{g}_{0}$ and then laser output power in the axial region of medium and large tubes is related to the reduction of temperature in this region, and more reduction of them in near-wall region for all size of tubes is due to minor amplification in this area, which is related to unsuitable electron temperature for forming of desirable population inversion $\left(\Delta \mathrm{N}_{0}\right)$, so.

The tube wall temperature is kept constant (here $\sim 510^{\circ} \mathrm{C}$ ) during the experiments, and then, the stimulated emission cross section, $\left(\sigma_{\mathrm{st}}\right)$ is approximately remain constant. Therefore, the $\mathrm{g}_{0}=\sigma_{\mathrm{st}} \Delta \mathrm{N}_{0}$, may be only dependent on $\Delta \mathrm{N}_{0}$. On the other hand, the temperature of electrons, which determined the optimum $\Delta \mathrm{N}_{0}$ is not constant through the tube crosssection. Therefore, the radial gain distribution is depended on tube bore, whereas, we show that the gain is not depended on length of tube, before (S. M. Lima et al., 2017).

The axial dip of the gain in medium and large tube bores can be due to the different efficiencies of excitation of the resonant and metastable levels because of increasing electron concentration during the pumping pulses, or due to the different velocities of volumetrically and near-wall mechanisms of plasma relaxation. The over-heating at the axis, the radial inhomogeneity of electric field, and skin effect can also contribute to the radial inhomogeneity (Fedor Aleksandrovich Gubarev \& Klenovskii, 2016). However, it seems that the above- mentioned mechanisms have less effect on gain profile in small-bore tubes. Therefore, we see that the gain profile of small- bore tube can be flatter and close to the Gaussian's shape.

In our previous works, a semi- experimental method was used for measuring the plasma temperature and obtaining the temperature profile of CVL and CBL (S Behrouzinia \& Khorasani, 2016; S. M. Lima et al., 2017; Namnabat, Behrouzinia, Moradi, \& Khorasani, 2016). The temperature of the center region is higher than that of the tube wall. At temperature above that of the optimum value in the center region, $\sigma_{\text {st }}$ increases by a little value, through increasing of Doppler width proportional to $\mathrm{T}^{1 / 2}$, and the Doppler-broadened line shape, so, but $\Delta \mathrm{N}_{0}$ decreased mainly due to inelastic collisions. In this case, $\Delta \mathrm{N}_{0}$ has a dominant effect relative to $\sigma_{\mathrm{st}}$.

The result indicates the gain will decreased (S. M. Lima et al., 2017). At optimum temperature, $\mathrm{g}_{0}$ and the gain have highest value, which this occurs between axial and wall regions in large and medium bore tubes, and at center of small one. At temperature lower than that of optimum value, the gain is dropped, especially at near the wall. Therefore, the gain radial distribution is depended on local temperature, and has a similar behavior to radial temperature profile. Also, the gain radial distributions is shown in Fig.4 for all tubes. 


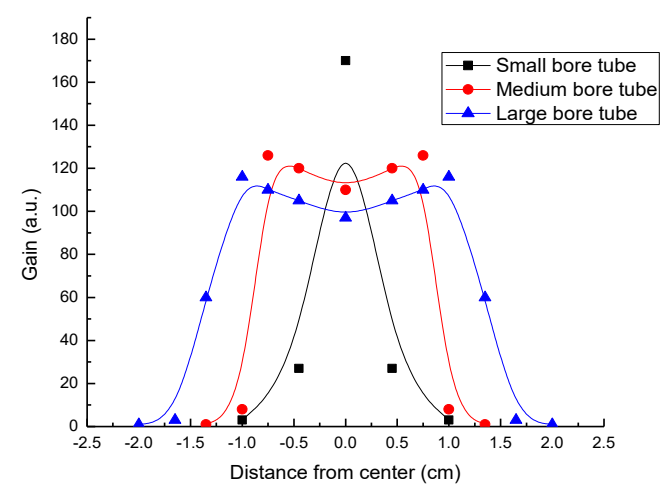

Fig.4. Gain radial distributions in different bore of tubes.

\section{CONCLUSIONS}

The experimental results shows that, as the tube bores of CBLs increases, the gain profile of the active medium becomes

\section{REFERENCES}

Behrouzinia, S., \& Khorasani, K. (2016). Frequency dependence of the temperature profile of a copper vapor laser. Laser Physics, 26(3), 035002.

Behrouzinia, S., Khorasani, K., Kazemi, H., \& Mashayekhi, H. (2011). Frequency dependence of amplifying parameters of a copper vapor laser using air as a buffer gas. Journal of Russian Laser Research, 32(6), 511-517.

Behrouzinia, S., Sadighi-Bonabi, R., Parvin, P., \& Zand, M. (2004). Temperature dependence of the amplifying parameters of a copper vapor laser. LASER PHYSICSLAWRENCE-, 14(8), 1050-1053.

Behrouzinia, S., Sadighi, R., \& Parvin, P. (2003). Pressure dependence of the small-signal gain and saturation intensity of a copper vapor laser. Applied optics, 42(6), 1013-1018.

Evtushenko, G. S., Torgaev, S.N., Trigub, M.V., Shiyanov, D.V., Evtushenko, T.G., Kulagin, A.E. . (2015). Opt. Commun. (338), 322.

Gubarev, F., Li, L., Klenovskii, M., \& Shiyanov, D. (2016). Spatial-temporal gain distribution of a $\mathrm{CuBr}$ vapor brightness amplifier. Applied Physics B, 122(11), 284.

Gubarev, F. A., \& Klenovskii, M. (2016). A long pulse $\mathrm{CuBr}$ vapor oscillator for laser monitor applications. Paper presented at the Journal of Physics: Conference Series.

Gubarev, F. A., Trigub, M. V., Klenovskii, M., Li, L., \& Evtushenko, G. S. (2016). Radial distribution of radiation in a $\mathrm{CuBr}$ vapor brightness amplifier used in laser monitors. Applied Physics B, 122(1), 2.

Gubarev, F. A., Troitskiy, V., Trigub, M. V., \& Sukhanov, V. B. (2011). Gain characteristics of large volume $\mathrm{CuBr}$ laser wider, consistent with the gain factor decrease in the axial region of discharge tube. The gain profile in small bore tube, has a Gaussian shape, but in medium bore tube, has a dip in axial region and in large one, the more dip has been occurred in axial region. It should be noted that, the radial average of gains are identical in all tube bores $\left(0.07 \mathrm{~cm}^{-1}\right)$. Before, it was demonstrated that the $\mathrm{g}_{0}$ is independent on laser tube length, but now, in this work, we show that the $\mathrm{g}_{0}$ is dependent on laser tube bore, locally. However, the average of radial gains are the same in different laser tube bores. Therefore, we can say that, the small-signal gain and gain of CBLs (probably CVLs and other gas lasers) is independent on geometrical factors of laser tube. The inhomogeneity of gain profile is related to inhomogeneity of temperature through the cross- section of laser tube.

\section{ACKNOWLEDGEMENTS}

The author gratefully acknowledge K. Khorasani and M. Fathalian for their interest on this work and helpful cooperation.

active media. Optics Communications, 284(10-11), 25652568.

Kulagin, A., Torgaev, S., \& Evtushenko, G. (2020). Kinetic modeling of amplifying characteristics of copper vapor active media for a wide range of input radiation power. Optics Communications, 460, 125136.

Li, L., Ilyin, A., Gubarev, F., Mostovshchikov, A., \& Klenovskii, M. (2018). Study of self-propagating hightemperature synthesis of aluminium nitride using a laser monitor. Ceramics International, 44(16), 19800-19808.

Lima, S. M., Behrouzinia, S., \& Khorasani, K. (2019). Amplifying characteristics of small-bore copper bromide lasers. Applied Physics B, 125(6), 101.

Lima, S. M., Behrouzinia, S., Salem, M., Elahei, M., Khorasani, K., \& Dorranian, D. (2017). The behavior of gain and saturation characteristics versus temperature in a copper bromide laser. Laser Physics, 27(5), 055001.

Lima, S. M., Behrouzinia, S., Salem, M.K., Elahei, M., Khorasani, K.,Dorranian, D. . ((2017)). Opt. Quantum Electron, 49, 372.

Namnabat, M., Behrouzinia, S., Moradi, A., \& Khorasani, K. (2016). Determination of plasma temperature of copper vapour laser. Journal of Plasma Physics, 82(1).

Nezhad, M. A., Sajad, B., Behrouzinia, S., Salehinia, D., \& Khorasani, K. (2010). Pressure dependence of small signal gain and saturation intensity of a gold-vapor laser using various buffer gases in gain medium. Optics Communications, 283(7), 1386-1388.

Trigub, M., Platonov, V., Evtushenko, G., Osipov, V., \& Evtushenko, T. (2017). Laser monitors for high speed imaging of materials modification and production. Vacuum, $143,486-490$. 